\title{
Tibial aplasia-ectrodactyly syndrome
}

\author{
INSERM
}

\section{Source}

INSERM. (1999). Orphanet: an online rare disease and orphan drug data base. Tibial aplasia-ectrodactyly syndrome. ORPHA:3329

Tibial aplasia-ectrodactyly syndrome is a rare condition characterized by congenital ectrodactylous limb malformations associated with tibial aplasia or hypoplasia. 